\section{Computation of Minimal Periodic Realizations of Transfer-Function Matrices}

\author{
Andras Varga
}

\begin{abstract}
We present a numerical approach to compute a minimal periodic state-space realization of a transfer-function matrix corresponding to a lifted state-space representation. The proposed method determines a realization with time-varying state dimensions by using exclusively orthogonal transformations. The new method is numerically reliable, computationally efficient and thus well suited for robust software implementations.
\end{abstract}

Index Terms-Discrete-time systems, minimal realization, numerical methods, periodic systems, time-varying systems.

\section{INTRODUCTION}

We consider the development of an efficient and reliable numerical algorithm for the following periodic realization problem (PRP): Given a $N p \times N m$ transfer-function matrix (TFM) $W(z)$, determine a minimal periodic realization (i.e., completely reachable and completely observable) of the form

$$
\begin{aligned}
x(k+1) & =A_{k} x(k)+B_{k} u(k) \\
y(k) & =C_{k} x(k)+D_{k} u(k)
\end{aligned}
$$

such that the TFM of the standard lifted representation of (1) (see Section II) is equal to $W(z)$. In (1), $A_{k} \in \mathrm{R}^{n_{k+1} \times n_{k}}, B_{k} \in \mathrm{R}^{n_{k+1} \times m}$, $C_{k} \in \mathrm{R}^{p \times n_{k}}, D_{k} \in \mathrm{R}^{p \times m}$ are periodic matrices with period $N \geq 1$. Note that, generally, the minimal periodic realization of a given $W(z)$ is a periodic system with time-varying state dimensions [1].

A computational algorithm to solve the above PRP is useful in many applications. For example, using lifted representations of periodic systems (as those introduced in [2], [5], [8], and [9]), it is relatively easy to compute left or right inverses of a periodic system by manipulating the associated system pencil matrix (see, for example, [11]). However, from the resulting representation of the inverse it is impossible in general to directly recover the underlying periodic representation. A minimal realization algorithm for periodic systems can be useful by allowing the following computational detour: compute first the corresponding TFM or a minimal state-space representation of the inverse and then compute its minimal periodic realization.

Another possible application is in the identification of periodic systems. For example, one of proposed subspace identification algorithms [14] is only applicable provided the underlying periodic system is uniformly reachable and observable (i.e., the periodic system has constant state dimension). This condition is however not always fulfilled, a notable exception being the class of multirate systems modeled as periodic systems. In this case, a computational detour is to identify first the TFM or state-space realization of the lifted system by using appropriate subspace identification methods, and then convert this model into a periodic minimal realization.

Manuscript received January 21, 2003; revised June 27, 2003. Recommended by Associate Editor D. E. Miller.

The author is with the German Aerospace Center, DLR-Oberpfaffenhofen, Institute of Robotics and Mechatronics, D-82234 Wessling, Germany (e-mail: andras.varga@dlr.de).

Digital Object Identifier 10.1109/TAC.2003.821421
Finally, the TFM to state-space conversion is a useful transformation which must belong to any software toolbox devoted to the manipulation of periodic system descriptions. Note that for the reverse transformation (the state-space to TFM conversion), a numerically reliable algorithm has been recently developed in [13].

A realization algorithm for periodic systems has been proposed by Lin and King [7]. The resulting periodic realization is however not minimal, because the state dimension is forcedly chosen constant. The determination of this state dimension requires checking of $N-1$ rank conditions. To compute a minimal realization, we need to combine this algorithm with a minimal realization algorithm, as for instance, that proposed in [10]. Besides the higher computational costs of the overall approach, both main computational steps use nonorthogonal transformations. Thus, in general, this combination approach is numerically not satisfactory.

In this note, we propose a computational procedure which improves the algorithm of [7] in two directions. First, the new procedure computes directly a periodic minimal realization with time-varying state dimensions starting from a minimal realization of $W(z)$ as a standard state-space system. Second, the procedure relies exclusively on performing orthogonal transformations, thus is completely satisfactory from numerical point of view. Therefore, the new method is well suited for robust software implementations.

\section{Periodic Realization PROBlem}

First, we introduce some notations and recall the definitions of reachability, observability and minimality of periodic systems (see [1] and [3]). The transition matrix of the system (1) is defined by the $n_{j} \times n_{i}$ matrix $\Phi_{A}(j, i)=A_{j-1} A_{j-2} \ldots A_{i}$, where $\Phi_{A}(i, i):=I_{n_{i}}$. The state transition matrix over one period $\Phi_{A}(j+N, j) \in \mathrm{R}^{n_{j} \times n_{j}}$ is called the monodromy matrix of system (1) at time $j$ and its eigenvalues are called characteristic multipliers at time $j$.

Definition 1: The periodic system (1) is reachable at time $i$ if

$$
\operatorname{rank} \mathcal{R}_{i}=n_{i}
$$

where $\mathcal{R}_{i}$ is the infinite columns matrix

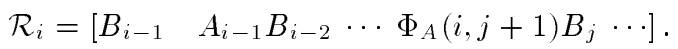

The periodic system (1) is completely reachable if (2) holds for $i=$ $1, \ldots, N$.

Definition 2: The periodic system (1) is observable at time $i$ if

$$
\operatorname{rank} \mathcal{O}_{i}=n_{i}
$$

where $\mathcal{O}_{i}$ is the infinite rows matrix

$$
\mathcal{O}_{i}=\left[\begin{array}{c}
C_{i} \\
C_{i+1} A_{i} \\
\vdots \\
C_{j} \Phi_{A}(j, i) \\
\vdots
\end{array}\right] .
$$

The periodic system (1) is completely observable if (4) holds for $i=$ $1, \ldots, N$.

Definition 3: The periodic system (1) is minimal if it is completely reachable and completely observable.

To define the TFM of the periodic system (1), we consider the timeinvariant representation corresponding to the associated lifted system 
introduced in [8] which uses the input-output behavior of the system over time intervals of length $N$, rather than 1 . For a given sampling time $k$, the corresponding $N m$-dimensional input, $N p$-dimensional output, and $n_{k}$-dimensional state vectors are defined as

$$
\begin{aligned}
& \widetilde{u}_{k}(h)=\left[u^{T}(k+h N) \cdots u^{T}(k+h N+N-1)\right]^{T} \\
& \widetilde{y}_{k}(h)=\left[y^{T}(k+h N) \cdots y^{T}(k+h N+N-1)\right]^{T} \\
& \widetilde{x}_{k}(h)=x(k+h N) .
\end{aligned}
$$

The lifted system has the form

$$
\begin{aligned}
\widetilde{x}_{k}(h+1) & =F_{k} \widetilde{x}_{k}(h)+G_{k} \widetilde{u}_{k}(h) \\
\widetilde{y}_{k}(h) & =H_{k} \widetilde{x}_{k}(h)+L_{k} \widetilde{u}_{k}(h)
\end{aligned}
$$

where

$$
\begin{aligned}
F_{k}= & \Phi_{A}(k+N, k) \\
G_{k}= & {\left[\Phi_{A}(k+N, k+1) B_{k} \cdots B_{k+N-1}\right] } \\
H_{k}= & {\left[\begin{array}{c}
C_{k} \\
C_{k+1} \Phi_{A}(k+1, k) \\
\vdots \\
C_{k+N-1} \Phi_{A}(k+N-1, k)
\end{array}\right] } \\
L_{k}= & {\left[\begin{array}{cccc}
D_{k} & 0 & \cdots & 0 \\
L_{k, 2,1} & D_{k+1} & \cdots & 0 \\
\vdots & \vdots & \ddots & \vdots \\
L_{k, N, 1} & L_{k, N, 2} & \cdots & D_{k+N-1}
\end{array}\right] }
\end{aligned}
$$

with $L_{k, i, j}=C_{k+i-1} \Phi_{A}(k+i-1, k+j) B_{k+j-1}$, for $i=2, \ldots, N$, $j=1,2, \ldots N-1$, and $i>j$.

The system (7) is called the standard lifted system at time $k$ of the given $N$-periodic system (1). The lifted system (7) shares the same structural properties as the original periodic system (1). In particular, the system (7) is reachable (observable) if and only if the system (7) is reachable (observable) at time $k$.

The associated TFM $W_{k}(z)$ is

$$
W_{k}(z)=H_{k}\left(z I_{n k}-F_{k}\right)^{-1} G_{k}+L_{k}
$$

and depends on the sampling time $k$. Thus, a given TFM $W(z)$ can be realized in $N$ instances as a periodic system.

In what follows, we assume that the given $N p \times N m$ TFM $W(z)$ corresponds to the time moment $k=1$ for which reason we will drop the index $k$. Realizations at time moments $k>1$ can be easily obtained by cyclic permutations of the matrices determined for $k=1$. We have the following result showing the existence of periodic realizations [6]:

Theorem 1: The $N p \times N m$ proper TFM $W(z)$ has a periodic realization of the form (1) if and only if the $W(\infty)$ matrix with $p \times m$ diagonal blocks is lower block triangular.

For our developments, we assume in what follows that $W(z)$ fulfills the condition of Theorem 1 and has a minimal realization of order $\bar{n}$, as a standard system $(\bar{A}, \bar{B}, \bar{C}, \bar{D})$ satisfying

$$
W(z)=\bar{C}\left(z I_{\bar{n}}-\bar{A}\right)^{-1} \bar{B}+\bar{D} .
$$

To solve the PRP we have to compute the $N$-periodic system matrices $A_{k}, B_{k}, C_{k}$, and $D_{k}$ which satisfy the conditions

$$
\bar{A}=F \quad \bar{B}=G \quad \bar{C}=H \quad \bar{D}=L .
$$

Moreover, the periodic realization $\left(A_{k}, B_{k}, C_{k}, D_{k}\right)$ is required to be minimal, that is, completely reachable and completely observable.

\section{PERIOdic REALIZATION Algorithm}

If we partition the matrices $\bar{B}, \bar{C}$, and $\bar{D}$ to correspond to the $N$ block rows and $N$ block columns of $W(z)$, we have

$$
\begin{aligned}
& \bar{B}=\left[\begin{array}{llll}
\bar{B}_{1} & \bar{B}_{2} & \cdots & \bar{B}_{N}
\end{array}\right] \\
& \bar{C}=\left[\begin{array}{c}
\bar{C}_{1} \\
\bar{C}_{2} \\
\vdots \\
\bar{C}_{N}
\end{array}\right] \quad \bar{D}=\left[\begin{array}{cccc}
\bar{D}_{11} & 0 & \cdots & 0 \\
\bar{D}_{21} & \bar{D}_{22} & \cdots & 0 \\
\vdots & \vdots & \ddots & \vdots \\
\bar{D}_{N 1} & \bar{D}_{N 2} & \cdots & \bar{D}_{N N}
\end{array}\right] \text {. }
\end{aligned}
$$

From (10), we get the following equations to be satisfied by the periodic system matrices $A_{k}, B_{k}, C_{k}$, and $D_{k}$

$$
\begin{aligned}
\bar{A} & =\Phi_{A}(N+1,1) \\
\bar{B}_{i} & =\Phi_{A}(N+1, i+1) B_{i}, \quad i=1, \ldots, N \\
\bar{C}_{i} & =C_{i} \Phi_{A}(i, 1), \quad i=1, \ldots, N \\
\bar{D}_{i i} & =D_{i}, \quad i=1, \ldots, N \\
\bar{D}_{i j} & =C_{i} \Phi_{A}(i, j+1) B_{j}, \quad 1 \leq j<i \leq N .
\end{aligned}
$$

We solve the previous equations by generating recursively the system matrices. We have immediately, that

$$
B_{N}=\bar{B}_{N} \quad C_{1}=\bar{C}_{1} \quad D_{i}=\bar{D}_{i i} \quad i=1, \ldots, N
$$

and, thus

$$
n_{1}=\bar{n}
$$

Analogously to [7], we consider the $(\bar{n}+(N-i) p) \times(\bar{n}+m i)$ matrices for $i=1, \ldots, N-1$

$$
K_{i}=\left[\begin{array}{c|ccc}
\bar{A} & \bar{B}_{1} & \cdots & \bar{B}_{i} \\
\hline \bar{C}_{N} & \bar{D}_{N, 1} & \cdots & \bar{D}_{N, i} \\
\vdots & \vdots & \ddots & \vdots \\
\bar{C}_{i+1} & \bar{D}_{i+1,1} & \vdots & \bar{D}_{i+1, i}
\end{array}\right]
$$

which can be factored in the form $K_{i}=K_{i}^{C} K_{i}^{B}$, where

$$
\begin{aligned}
K_{i}^{C} & =\left[\begin{array}{c}
\frac{\Phi_{A}(N+1, i+1)}{C_{N} \Phi_{A}(N, i+1)} \\
\vdots \\
C_{i+1}
\end{array}\right] \\
K_{i}^{B} & =\left[\Phi_{A}(i+1,1) \mid \Phi_{A}(i+1,2) B_{1} \cdots B_{i}\right] .
\end{aligned}
$$

Let

$$
n_{i+1}:=\operatorname{rank} K_{i}
$$

and consider the full rank orthogonal factorization

$$
K_{i}=U_{i} R_{i}
$$

with the $(\bar{n}+(N-i) p) \times n_{i+1}$ matrix $U_{i}$ having orthonormal columns (i.e., $\left.U_{i}^{T} U_{i}=I_{n_{i+1}}\right)$, and the $n_{i+1} \times(\bar{n}+m i)$ matrix $R_{i}$ having full row rank. We partition $U_{i}$ and $R_{i}$ compatibly as follows:

$$
U_{i}=\left[\frac{\bar{U}_{i, 1}}{\bar{U}_{i, 2}}\right] \quad R_{i}=\left|\bar{R}_{i, 1}\right| \bar{R}_{i, 2} \mid
$$


where $\bar{U}_{i, 2}$ and $\bar{R}_{i, 2}$ are $p \times n_{i+1}$ and $n_{i+1} \times m$ matrices, respectively. We can immediately take

$$
B_{i}=\bar{R}_{i, 2} \quad C_{i+1}=\bar{U}_{i, 2} .
$$

If $i=1$, we can choose

$$
A_{i}=\bar{R}_{1,1}
$$

while for $i=N-1$ we can choose

$$
A_{N}=\bar{U}_{N-1,1} \text {. }
$$

For $i=2, \ldots, N-1$, it is easy to see that $A_{i}$ satisfies

$$
U_{i} A_{i}=\bar{U}_{i-1,1} .
$$

Provided range $\left(\bar{U}_{i-1,1}\right) \subseteq \operatorname{range}\left(U_{i}\right)$, and recalling that $U_{i}$ has full column rank and orthonormal columns, we get the unique solution

$$
A_{i}=U_{i}^{T} \bar{U}_{i-1,1} \text {. }
$$

To show that indeed range $\left(\bar{U}_{i-1,1}\right) \subseteq \operatorname{range}\left(U_{i}\right)$, we observe that $K_{i}$ and $K_{i-1}$ can be partitioned as

$$
\begin{aligned}
& K_{i}=\left[\begin{array}{l|c} 
& \bar{B}_{i} \\
X_{i} & \bar{D}_{N, i} \\
& \vdots \\
& \bar{D}_{i+1, i}
\end{array}\right] \\
& K_{i-1}=\left[\frac{X_{i}}{\bar{C}_{i} \bar{D}_{i, 1} \cdots \bar{D}_{i, i-1}}\right] \text {. }
\end{aligned}
$$

Taking into account the partitioning of $U_{i-1}$ in the form

$$
U_{i-1}=\left[\frac{\bar{U}_{i-1,1}}{\bar{U}_{i-1,2}}\right]
$$

it follows that range $\left(\bar{U}_{i-1,1}\right)=\operatorname{range}\left(X_{i}\right) \subseteq \operatorname{range}\left(K_{i}\right)=$ range $\left(U_{i}\right)$.

We can easily prove our main result.

Theorem 2: The periodic realization computed by using the formulas (12)-(19) is minimal.

Proof: We need to show that for the computed system matrices satisfying the equations (11), the corresponding state dimensions (13) and (14) satisfy

$$
n_{i}=\operatorname{rank} \mathcal{R}_{i}=\operatorname{rank} \mathcal{O}_{i}, \quad i=1, \ldots, N .
$$

First, we show that

$$
\operatorname{rank} \mathcal{R}_{1}=n_{1}
$$

where

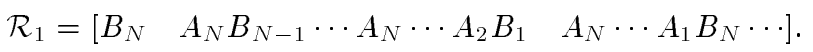

Since the pair $(\bar{A}, \bar{B})$ is reachable, we have that

$$
n_{1}=\bar{n}=\operatorname{rank}\left[\bar{B} \quad \overline{A B} \cdots \bar{A}^{\bar{n}-1} \bar{B}\right]=\operatorname{rank} \mathcal{R}_{1} .
$$

Similarly, from the observability of the pair $(\bar{A}, \bar{C})$, it follows that

$$
n_{1}=\operatorname{rank} \mathcal{O}_{1}
$$

By denoting

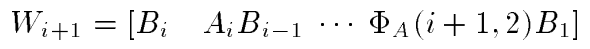

we can partition $\mathcal{R}_{i+1}$, for $i \geq 1$, as

$$
\mathcal{R}_{i+1}=\left[W_{i+1} \mid \Phi_{A}(i+1,1) \mathcal{R}_{1}\right] .
$$

From the full rank factorization (15), we have that

$$
\operatorname{rank}\left[W_{i+1} \mid \Phi_{A}(i+1,1)\right]=n_{i+1} .
$$

Since $\mathcal{R}_{1}$ has full row rank, it is easy to show that

$$
\operatorname{rank}\left[W_{i+1} \mid \Phi_{A}(i+1,1) \mathcal{R}_{1}\right]=\operatorname{rank}\left[W_{i+1} \mid \Phi_{A}(i+1,1)\right]
$$

and, thus

$$
\operatorname{rank} \mathcal{R}_{i+1}=n_{i+1} \text {. }
$$

Similarly, we can prove the observability properties.

There are two main improvements offered by our algorithm with respect to the algorithm of [7]. The first improvement is that we determine directly a periodic realization with minimal order state dimensions. The resulting periodic realization is minimal and has, in general, time-varying state dimensions. In contrast, the algorithm of [7] determines generally a nonminimal realization with unreachable and/or unobservable characteristic multipliers in the origin.

The second improvement is the overall numerically reliability of our algorithm and the straightforward computation of the state matrices of the periodic realization. The computation of the minimal periodic realization involves performing $N-1$ rank revealing QR-factorizations (e.g., QR-factorizations with column pivoting or singular value decompositions [4]) and all matrices are generated in terms of the computed quantities in these factorizations. By using the orthogonal full rank factorizations (15), we can explicitly solve the equation $U_{i} A_{i}=\bar{U}_{i-1,1}$ satisfied by $A_{i}$. Thus all system matrices can be determined by using exclusively orthogonal transformations. In contrast, the algorithm of [7] determine $A_{i}$ by solving two matrix equations involving both $U_{i}$ and $R_{i}$ (see [7, Lemma 2.1]). Thus, our algorithm can be considered completely satisfactory from numerical point of view along the lines of requirements formulated in [12].

We can roughly estimate the computational effort required by our algorithm assuming constant dimension $n_{i}=n, i=1, \ldots, N$ for the resulting periodic realization. We assume that we use QR-decompositions with column pivoting based on Householder transformations to compute the full rank orthogonal factorizations (see [4] for details). Note that for a generic $m \times n$ matrix $X$ of rank $r$, the computation of the $r \times n$ matrix $R$ in the full rank QR factorization $X=$ $Q R$ requires about $r\left(2 m n+\left(2 r^{2} / 3\right)-(m+n) r\right)$ flops $(1$ flop $=$ 1 multiplication +1 addition), while the economic accumulation of the $r$ Householder transformations in $Q$ requires $r^{2}(2 m-r)$ flops. Thus, in total we need about $r\left(2 m n-\left(r^{2} / 3\right)+(m-n) r\right)$ flops. In the minimal realization algorithm we need to compute $N-1$ full rank factorizations of the $K_{i}$ matrices of dimensions $(\bar{n}+(N-i) p) \times(\bar{n}+i m)$, for $i=1, \ldots, N-1$. By assuming $n \approx \bar{n}$, this involves approximately

$$
n(N-1)\left(\frac{5 n^{2}}{3}+\frac{(3 p+m) n N}{2}+\frac{p m N^{2}}{3}\right)
$$

flops. To the above estimation we have to add the number of flops necessary to compute the state-space realization of $W(z)$. 


\section{EXAMPLE}

We consider the example used in [7] to show the main computational steps. For the sake of clarity, we will use nonorthogonal computations to compute the full rank factorizations. Let

$$
W(z)=\frac{1}{z-1}\left[\begin{array}{ccc}
z+2 & 4 & 1 \\
6 z & 3 z+5 & 2 \\
9 z & z+11 & z+2
\end{array}\right]
$$

and consider the same state-space realization as in [7]

$$
\left[\begin{array}{l|l}
\bar{A} & \bar{B} \\
\hline \bar{C} & \bar{D}
\end{array}\right]=\left[\begin{array}{l|lll}
1 & 3 & 4 & 1 \\
\hline 1 & 1 & 0 & 0 \\
2 & 6 & 3 & 0 \\
3 & 9 & 1 & 1
\end{array}\right] .
$$

We have immediately

$$
B_{3}=1 \quad C_{1}=1 \quad D_{1}=1 \quad D_{2}=3 \quad D_{3}=1 .
$$

From

$$
K_{1}=\left[\begin{array}{ll}
1 & 3 \\
3 & 9 \\
2 & 6
\end{array}\right]=\left[\begin{array}{l}
1 \\
\frac{3}{2}
\end{array}\right][1 \mid 3]
$$

we obtain

$$
A_{1}=1 \quad B_{1}=3 \quad C_{2}=2 .
$$

Further, from

$$
K_{2}=\left[\begin{array}{lll}
1 & 3 & 4 \\
3 & 9 & 1
\end{array}\right]=\left[\begin{array}{ll}
1 & 4 \\
\hline 3 & 1
\end{array}\right]\left[\begin{array}{ll|l}
1 & 3 & 0 \\
0 & 0 & 1
\end{array}\right]
$$

we obtain

$$
B_{2}=\left[\begin{array}{l}
0 \\
1
\end{array}\right] \quad C_{3}=\left[\begin{array}{ll}
3 & 1
\end{array}\right] \quad A_{3}=\left[\begin{array}{ll}
1 & 4
\end{array}\right]
$$

$A_{2}$ results from

$$
\left[\begin{array}{ll}
1 & 4 \\
3 & 1
\end{array}\right] A_{2}=\left[\begin{array}{l}
1 \\
3
\end{array}\right]
$$

as

$$
A_{2}=\left[\begin{array}{l}
1 \\
0
\end{array}\right]
$$

The resulting periodic realization has state dimensions $n_{1}=1$, $n_{2}=1$ and $n_{3}=2$ and is minimal. In contrast, the realization obtained in [7] has constant order $n=2$ and is not minimal.

\section{CONCLUSION}

We proposed a numerically sound and computationally efficient approach to compute minimal periodic realizations of transfer-funtion matrices. The resulting periodic representations have in general timevarying dimensions. The proposed approach relies exclusively on numerically stable algorithms, the key computations being $N-1$ rank re- vealing orthogonal decompositions. The proposed approach is straightforward to implement as robust numerical software. Numerical examples computed with a MATLAB-based implementation show the applicability of this method to high order periodic systems.

\section{REFERENCES}

[1] P. Colaneri and S. Longhi, "The realization problem for linear periodic systems," Automatica, vol. 31, pp. 775-779, 1995

[2] D. S. Flamm, "A new shift-invariant representation of periodic linear systems," Syst. Control Lett., vol. 17, pp. 9-14, 1991.

[3] I. Gohberg, M. A. Kaashoek, and L. Lerer, "Minimality and realization of discrete time-varying systems," Operator Theory: Adv. Applicat., vol. 56, pp. 261-296, 1992.

[4] G. H. Golub and C. F. Van Loan, Matrix Computations. Baltimore, MD: John Hopkins Univ. Press, 1989.

[5] O. M. Grasselli and S. Longhi, "Finite zero structure of linear periodic discrete-time systems," Int. J. Syst. Sci., vol. 22, pp. 1785-1806, 1991.

[6] P. P. Khargonekar, K. Poola, and A. Tannenbaum, "Robust control of linear time-invariant plants using periodic compensation," IEEE Trans. Automat. Contr., vol. AC-30, pp. 1088-1096, Sept. 1985.

[7] C.-A. Lin and C.-W. King, "Minimal periodic realizations of transfer matrices," IEEE Trans. Automat. Contr., vol. 38, pp. 462-466, Mar. 1993.

[8] R. A. Meyer and C. S. Burrus, "A unified analysis of multirate and periodically time-varying digital filters," IEEE Trans. Circuits Syst., vol. CAS-22, pp. 162-168, 1975.

[9] B. Park and E. I. Verriest, "Canonical forms for discrete-time periodically time varying systems and a control application," in Proc. Conf. Decision Control, Tampa, FL, 1989, pp. 1220-1225.

[10] A. Varga, "Balancing related methods for minimal realization of periodic systems," Syst. Control Lett., vol. 36, pp. 339-349, 1999.

[11] — "Computing generalized inverse systems using matrix pencil methods," Int. J. Appl. Math. Comput. Sci., vol. 11, pp. 1055-1068, 2001.

[12] A. Varga and P. V. Dooren, "Computational methods for periodic systems-An overview," in Proc. IFAC Workshop Periodic Control Systems, Como, Italy, 2001, pp. 171-176.

[13] A. Varga, "Computation of transfer functions matrices of periodic systems," in Proc. Conf. Decision Control, Las Vegas, NV, 2002.

[14] M. Verhaegen and X. Yu, "A class of subspace model identification algorithms to identify periodically and arbitrarily time-varying systems," Automatica, vol. 31, pp. 201-216, 1995. 